\title{
Linfangioma quístico. Nuestra experiencia
}

\author{
Arzoz Fàbregas M, Ibarz Servio Ll, Areal Calama J, González Satué C, Ruiz Domínguez J, \\ Saladié Roig JM.
}

Servicio de Urología. Hospital Universitari Germans Trias I Pujol. Badalona. Barcelona.

Actas Urol Esp. 2006;30(7):723-727

\section{RESUMEN \\ LINFANGIOMA QUÍSTICO. NUESTRA EXPERIENCIA.}

Objetivo: Comentar los aspectos más relevantes y revisar la literatura de esta patología tan poco frecuente.

Material y métodos: Presentamos dos casos de linfangioma o higroma quístico, comentando la clínica, diagnóstico, tratamiento y la evolución de éstos y, revisando la literatura actual. El primer caso es el de un joven diagnosticado de linfangioma pélvico y escrotal. El segundo es el de un paciente con linfangioma quístico retroperitoneal.

Discusión: El linfangioma quístico es una malformación congénita hamartomatosa del sistema linfático que suele aparecer en la infancia a modo de grandes masas a nivel de partes blandas, con tendencia a crecer, a no ser que se extirpen de forma completa.

Conclusión: Son pocos los casos descritos de higroma quístico escrotal, muchas veces diagnosticados erróneamente, como otras patologías quísticas escrotales, lo que comporta indicaciones quirúrgicas erróneas y cirugias incompletas que predisponen a la recidiva posterior. El higroma quístico retroperitoneal aislado es una patología infrecuente diagnosticada a menudo de forma incidental.

Palabras clave: Linfangioma quístico. Escroto. Retroperitoneo.

\section{ABSTRACT \\ CYSTIC LYMPHANGIOMA. OUR EXPERIENCE}

Objective: To comment on the most relevant aspects and to review the literature on this unusual pathology.

Material and Methods: We present two cases of cystic lymphangioma, their clinical, diagnosis, therapy and development, and we review the actual literature. The first case is a young man with the diagnosis of cystic lymphangioma of the scrotum and pelvis. The second case is one patient with a cystic lymphangioma of the

retroperitoneum.

Discussion: Cystic lymphangioma is a congenital lymphatic hamartoma of the lymphatic system that usually appears at childhood like a big mass at soft tissues that has a tendency to grow unless a completed excision is performed.

Conclusions: There are few cases of scrotal cystic lymphangioma in the literature; they are often misdiagnosed as other common cystic scrotal conditions leading to an improper surgical approach and an incomplete surgery that predisposes later recurrence. Cystic lymphangioma of the retroperitoneum is a rare entity diagnosis in most cases as an incidentaloma. 
L os linfangiomas son malformaciones congénitas hamartomatosas del sistema linfático que afectan a la piel y al tejido subcutáneo. Se caracterizan por aparecer en la infancia a modo de grandes masas a nivel de partes blandas con tendencia a crecer a no ser que se extirpen de forma completa en sus etapas iniciales. El linfangioma quístico, también denominado higroma quístico, constituye una variante de linfangio$\mathrm{ma}^{1}$. Describimos dos casos de higroma quístico, de localización poco frecuente, controlados por el Servicio de Urología de nuestro hospital.

\section{CASOS CLÍNICOS}

Caso 1: Linfangioma quístico pélvico y escrotal.

Paciente de 21 años, diagnosticado hace diez años, por Resonancia Magnética Nuclear (RMN), de linfangioma quístico con afectación de hipogastrio, escroto y pelvis. Clínicamente presentaba un aumento progresivo del diámetro hipogástrico y escrotal con la bipedestación (Fig. 1). Como antecedentes destacaban dos intervenciones quirúrgicas en la infancia: una por hernia inguinal izquierda, y la otra por quiste de cordón izquierdo, ambas con recidiva postoperatoria inmediata. Se procedió a exéresis macroscópicamente completa del componente hipogástrico y escrotal, siendo el diagnóstico anatomopatológico de linfangioma quístico. A los dos años, es remitido a nuestro centro por presentar recidiva a nivel de área hipogástrica y escrotal. Se procede nuevamente a exéresis del componente linfangiomatoso de estas zonas, presentando otra recidiva a los cinco años del diagnóstico, por lo que se realiza una segunda reintervención. Durante estos últimos cinco años el paciente ha presentado un epi- sodio de linfangitis escrotal con buena respuesta a terapia con antibiótico endovenoso. En el último control, el paciente refiere sensación de hinchazón a nivel escrotal con la bipedestación, por lo que se realiza una ecografía escrotal en la que se evidencia la presencia de quistes a nivel intraescrotal e intratesticular izquierdo (Fig. 2). Se solicita posteriormente una RNM (Fig. 3) donde se confirma la presencia de quistes intratesticulares sin evidenciarse progresión del componente linfangiomatoso pélvico respeto a la primera RNM, por lo que se decide adoptar una conducta expectante.

Caso 2: Linfangioma quístico retroperitoneal

Paciente de 68 años con antecedentes de cinco episodios de neumonía y de prostatectomía radical

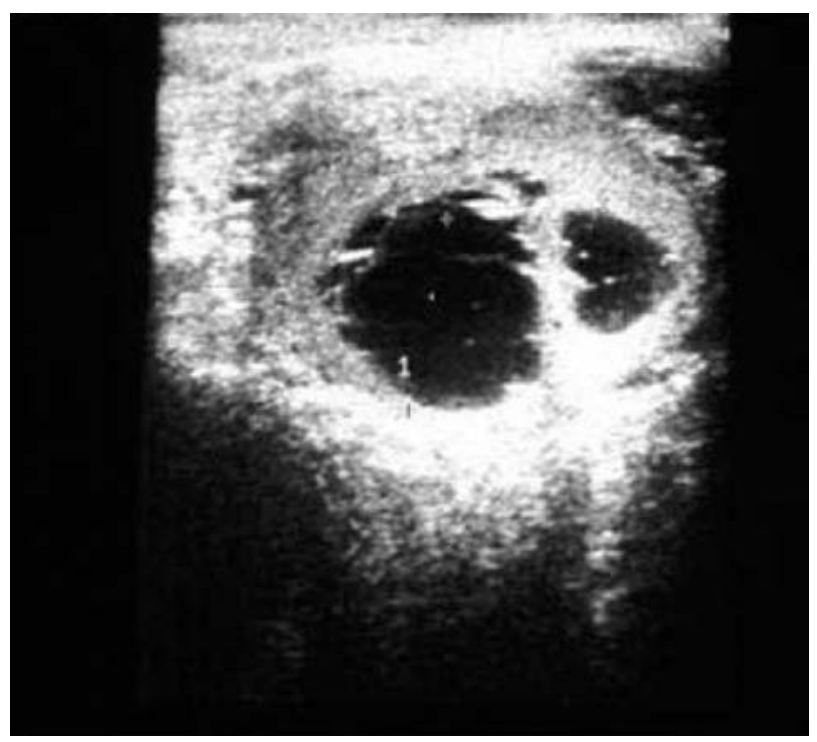

FIGURA 2. ECO escrotal: Quistes a nivel intratesticular.

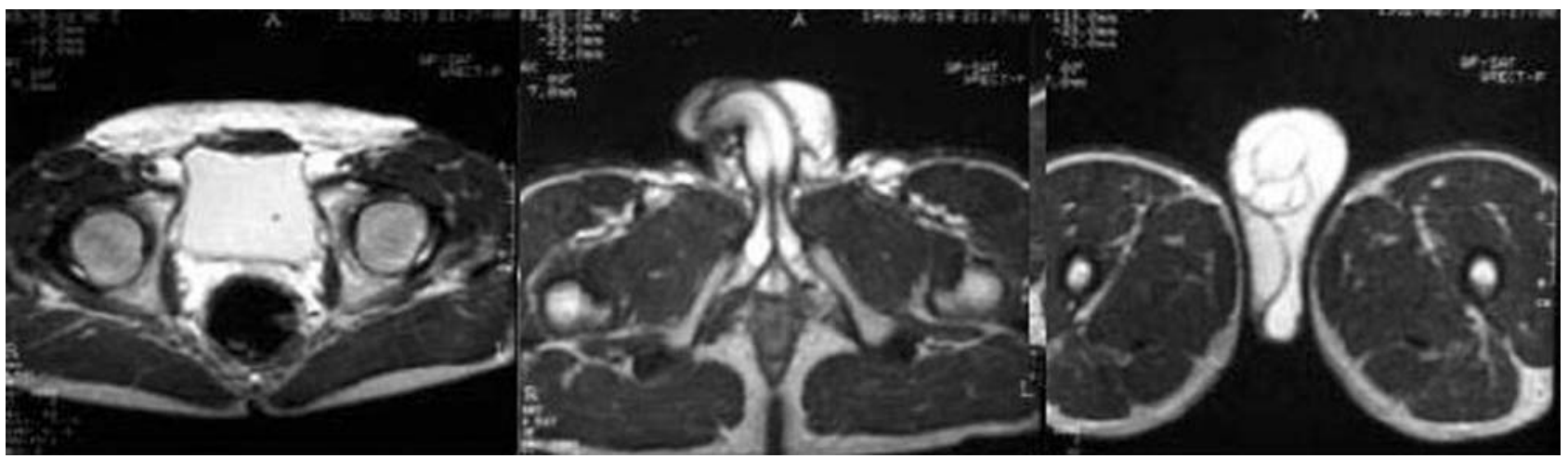

FIGURA 1. RNM pélvica: Linfangioma quístico a nivel de hipogastrio, pelvis y escroto. 


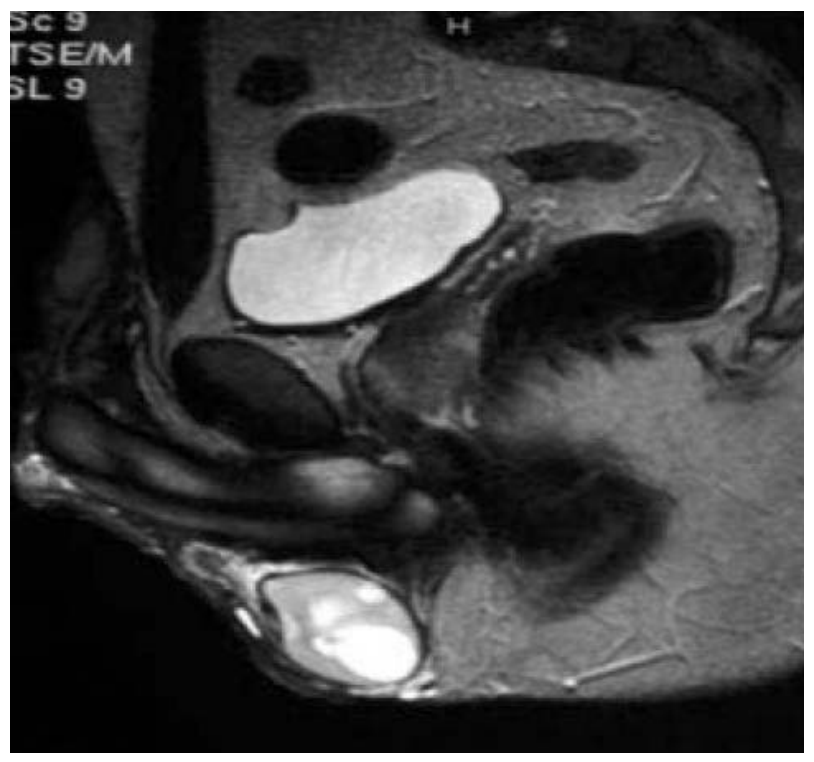

FIGURA 3. RNM pélvica: Quistes intratesticulares sin evidencia de progresión de componente linfangiomatoso a nivel pélvico.

hace 6 años, con recidiva local posterior y que requirió tratamiento con radioterapia. En el TAC de estudio de su enfermedad prostática se detecta una masa retroperitoneal hipointensa de unos $6 \times 2 \mathrm{cms}$ que desplaza la glándula suprarenal y el riñón izquierdo sin llegar a invadir dichas estructuras (Fig. 4). Se solicitó una RNM donde se evidenciaba una masa de características quísticas y morfología polilobulada a nivel retroperitoneal izquierdo sin invasión de estructuras retroperito-

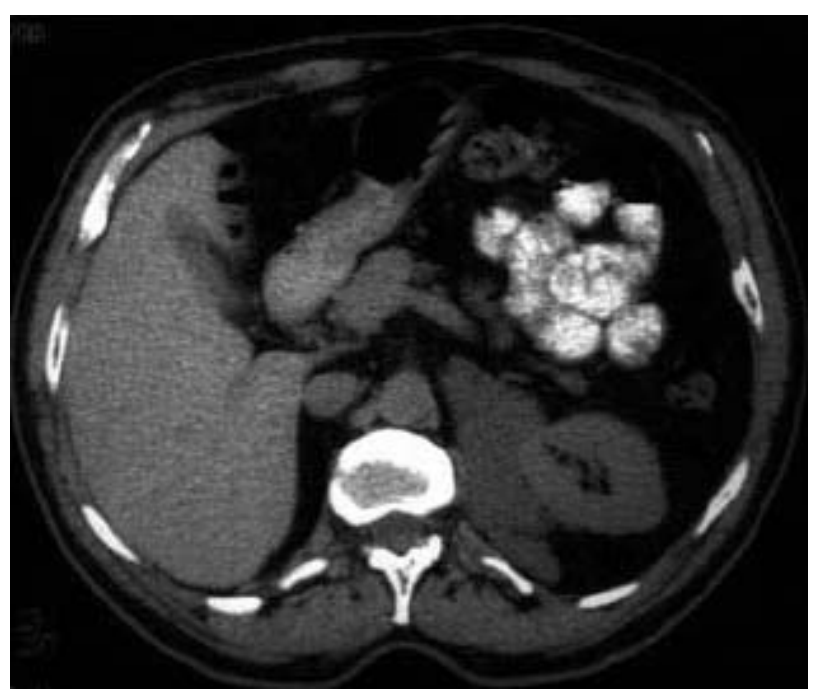

FIGURA 4. TAC abdominal: Masa retroperitoneal izquierda hipointensa que desplaza suprarrenal y riñón sin infiltrados. neales, compatible con linfangioma quístico. Ante ese diagnóstico se decidió adoptar una conducta expectante, alternando TAC y RNM para el control evolutivo. En último TAC (Fig. 5), no se observa progresión de la enfermedad.

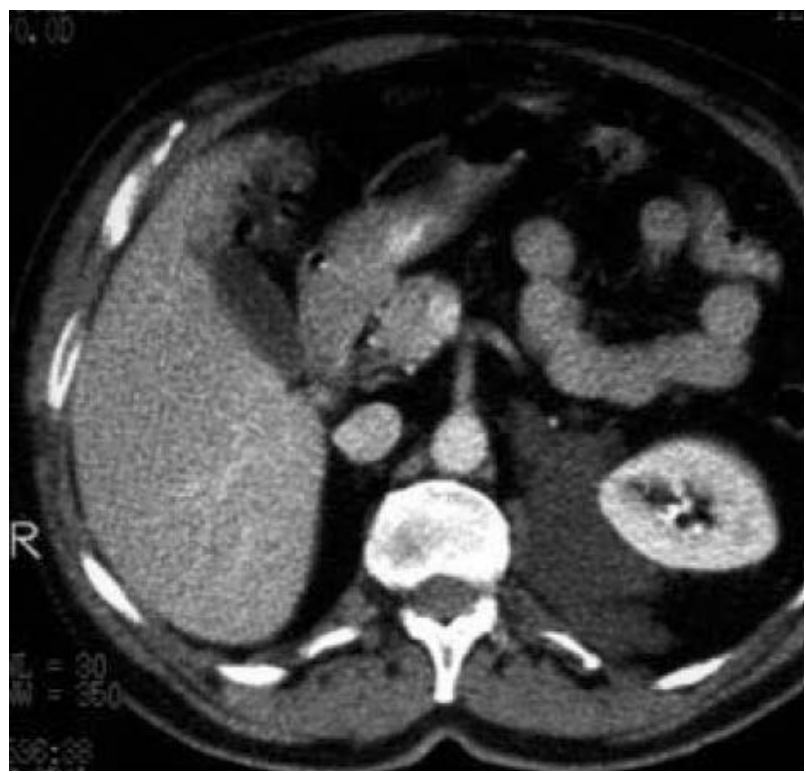

FIGURA 5. TAC abdominal de control. Sin progresión de la enfermedad.

\section{DISCUSIÓN}

Los linfangiomas son hamartomas congénitos del sistema linfático. Aunque de etiopatogenia poco clara, existe la hipótesis de que se debe a un insuficiente drenaje de los vasos linfáticos hacia el sistema venoso, secundario a la atresia o bien a la insuficiencia de los canales eferentes. Se clasifican en capilares, cavernosos o quísticos dependiendo de la profundidad y del tamaño de los vasos linfáticos afectados. La mitad están presentes en el momento del nacimiento y el $90 \%$ son evidentes antes de los dos años. No existen variaciones según el sexo ${ }^{1-3}$.

La localización más común de linfangioma es a nivel de la cabeza y el cuello. El higroma quístico aparece habitualmente en zonas de tejido areolar o tejido conectivo liso, frecuentemente en axila (75\%) y en cuello (20\%). Localizaciones menos habituales serían: el mediastino, área escrotal (Unos treinta casos descritos en la literatura) y retroperitoneo ${ }^{1,2}$. A pesar de ser consideradas lesiones sin potencial de malignidad, los linfangiomas suelen progresar, recidivar (sobre 
todo debido a cirugías incompletas previas) o complicarse por infecciones asociadas. El tratamiento de elección es la exéresis quirúrgica completa, aunque siempre debe ser conservadora pues la benignidad de estas lesiones no justifica el sacrificio de estructuras vitales ${ }^{1}$.

$\mathrm{El}$ ser conservadores comporta que muchas veces la cirugía sea incompleta y en consecuencia la recurrencia sea elevada. Es por ello que se han probado técnicas como la utilización de diatermia, la radioterapia y escleroterapia percutánea para el tratamiento de estas lesiones. De todas ellas, la escleroterapia es la que ha dado mejores resultados, aunque se han usado múltiples sustancias esclerosantes y aún se debate cual debe ser el agente de elección ${ }^{4,5}$.

Son pocos los casos descritos en la literatura de higroma quístico escrotal, aproximadamente unos treinta, muchas veces diagnosticados equivocadamente como otras patologias quísticas escrotales, lo que comporta indicaciones quirúrgicas erróneas y cirugías incompletas que predisponen a la recidiva posterior ${ }^{2,6}$. Se caracterizan por ser diagnosticados mayoritariamente en la infancia. El cuadro clínico más frecuente es el de inflamación escrotal no dolorosa. Muchas veces son confundidos con hernias, varicoceles $u$ otras patologías quísticas del cordón o escrotales, por lo que deberemos hacer el diagnóstico diferencial con estas entidades ${ }^{2}$. La ultrasonografía combinada con el Doppler es útil para confirmar el diagnóstico de sospecha clínico. En ocasiones, la afectación escrotal se asocia a una extensión perineal y/o retroperitoneal por lo que se aconseja la realización de un TAC o RNM (de elección en la infancia) ante la sospecha de esta entidad. Estas últimas exploraciones serán de gran utilidad durante el acto quirúrgico para poder planificar las zonas a extirpar ${ }^{6}$. El diagnóstico definitivo se obtiene a partir del examen anatomopatológico de la pieza quirúrgica.

En el primer caso, ejemplo que reafirma la importancia del diagnóstico inicial, se decidió realizar exéresis quirúrgica de las áreas sin repercusión a órganos vitales (por lo tanto incompleta); en las dos recidivas posteriores se optó por el mismo tratamiento. En la actualidad el paciente presenta mínima progresión a nivel escrotal y afectación intratesticular de novo sin progresión del componente pélvico. En esta última ocasión se optó por una actitud expectante.

A pesar de su rareza, la afectación linfangiomatosa intraescrotal es más frecuente a nivel del epidídimo que intratesticular. La afectación testicular se atribuye a la desestructuración de los túbulos seminiferos secundaria a la alteración de los vasos linfáticos a ese nivel ${ }^{7}$.

El higroma quístico retroperitoneal aislado es una patología infrecuente diagnosticada a menudo de forma incidental. La variedad capilar no afecta a este nivel. En ocasiones son sintomáticos debido a la compresión de estructuras vecinas, a episodios de infección o a episodios de hemorragia. El diagnóstico de esta entidad suele hacerse mediante pruebas de imagen, siendo los ultrasonidos, la resonancia y la tomografía las técnicas de elección ${ }^{3}$. La ecografía permite diferenciar la naturaleza de la lesión. La tomografía y la resonancia magnética nos dan información del tamaño, la extensión y la relación de la lesión respecto a las estructuras vecinas. La resonancia permite además detectar afectación ósea asociada, en los casos de linfangiomatosis múltiple, que podría pasar desapercibida con la tomografía o la radiografía simple ${ }^{3,8}$. Debemos hacer diagnóstico diferencial con patologías malignas del retroperitoneo como son: los tumores quísticos pancreáticos, las metástasis ganglionares retroperitoneales y los sarcomas retroperitoneales, así como de otras entidades benignas, ya sean hematomas, linfoceles secundarios a cirugías previas o abscesos, que pueden encontrarse a ese nivel. A pesar de que en la mayoría de la literatura se considera que el diagnóstico de confirmación se obtendrá a partir del examen anatomopatológico de la pieza quirúrgica, existen trabajos que sugieren que las alteraciones en el TAC y RNM contribuyen a un diagnóstico más preciso de esta patología y, que en ocasiones pueden ahorrar el uso de técnicas más invasivas o incluso de procedimientos quirúrgicos para llegar al diagnóstico de certeza $^{3,8}$.

En el segundo de nuestros casos, al estar el paciente asintomático desestimamos cualquier actitud terapéutica en el momento del diagnóstico. Durante los controles posteriores no se ha evidenciado progresión radiológica de la enfermedad y el paciente continua clínicamente estable. 


\section{CONCLUSIÓN}

Aunque poco frecuente, el linfangioma es una hamartomatosis benigna del sistema linfático que puede presentarse a nivel escrotal, pélvica y retroperitoneal; todas ellas áreas de implicación urológica. Es importante recordar esta patología e incluirla en el diagnóstico diferencial de las patologías quísticas con afectación escrotal y retroperitoneal, pues el éxito del tratamiento depende de un correcto diagnóstico inicial.

Se considera que el tratamiento de elección en los pacientes sintomáticos, ya sea con fines curativos, o estabilizadores, es la exéresis completa de la pieza quirúrgica. No debemos olvidar que se trata de una patología benigna y por ello, el tratamiento debe evitar en todo momento el sacrificio de estructuras vitales. Disponemos en la actualidad de métodos no quirúrgicos que aún están en fase de experimentación, de entre los cuales la escleroterapia percutánea es el que ha dado mejores resultados.

\section{REFERENCIAS}

1. Singh S, Baboo ML, Pathak IC. Cystic lymphangiomas in children: report of 32 cases including lesions at rare sites. Surgery. 1971;69(6):947-951
2. Hurwitz RS, Shapiro E, Hurbert WC, Diamond DA, Casale AJ, Rink RC. Scrotal Cystic Lymphangioma: The Misdiagnosed Scrotal Mass. J Urol 1997;158(3 Pt 2):1182-1185.

3. Bonhomme A, Broeders R, Oyen H, Stas M, de Wever, Baert AL. Cystic Lymphangioma of the retroperitoneum. Clin Radiol 2001; 56(2):156-158.

4. Fujino A, Moriya Y, Morikawa Y, Hoshino K, Watanabe T, Shimojima N, et al. A Role of Cytokines in OK-432 Injection Therapy for Cystic Lymphangioma: An Approach to the Mechanism. J Pediatr Surg 2003; 38(12):1806-1809.

5. Won JH, Kim BM, Kim C, Park SW, Kim MD. Percutaneous Sclerotherapy of Lymphangiomas with Acetic Acid. J Vasc Interv Radiol. 2004;15(6):595-600.

6. Loberant N, Chernihovski A, Goldfeld M, Sweed Y, Vais M, Tzilman B, et al. Role of Doppler Sonography in the diagnosis of Cystic Lymphangioma of the scrotum. J Clin Ultrasound 2002;30(6):384-387.

7. Garrett J.E, Cartwright PC, Snow BW, Coffin.M. Cystic testicular lesions in the pediatric population. J Urol 2000;163 (3):928-936.

8. Wunderbaldinger P, Paya K, Partik B, Turetschek K, Hormann M, Horcher E, et al. CT and MR imaging of Generalized Cystic Lymphangiomatosis in Pediatric Patients. AJR Am J Roentgenol. 2000 Mar; 174(3):827-832.

Dra. M. Arzoz Fábregas

E-mail: 37031maf@comb.es

(Trabajo recibido el 27 de abril 2005) 